\title{
NEOPLASMS
}

\section{NEUROCOGNITIVE DEFICITS IN MEDULLOBLASTOMA PATIENTS}

Eighteen children previously treated surgically and with craniospinal irradiation for medulloblastoma (MED), and matched with a group having lowgrade posterior fossa tumors with surgery alone, received neuropsychological testing and quantitative magnetic resonance imaging for white matter loss at St Jude Children's Research Hospital, Memphis, TN. Normal white matter (NWM) volume and Full Scale IQ values were significantly lower among survivors of MED compared to controls. Irradiation (with or without chemotherapy)-induced destruction of NWM may explain the intellectual and academic deficits associated with MED survival. (Mulhern RK, Reddick WE, Palmer SL et al. Neurocognitive deficits in medulloblastoma survivors and white matter loss. Ann Neurol Dec 1999;46:834-841). (Respond: Dr Raymond K Mulhern, St Jude Children's Research Hospital, 332 N Lauderdale, Memphis, TN 38105).

COMMENT. A lowered IQ in children treated for medulloblastoma is correlated with a loss of normal cerebral white matter. Diffuse and multifocal white matter hyperintensities demonstrated on MRI, and calcifications in cortical gray matter and basal ganglia have been correlated with the dose of cranial radiation therapy (CRT).

Younger patients and those receiving standard dose CRT are at greater risk of cognitive deficits than older children or those receiving reduced dose CRT (Mulhern RK et al. I Clin Oncol 1998;16:1723-1728). If dose of CRT cannot be lowered and white matter cannot be protected during irradiation treatment, children surviving MED should receive early cognitive intervention therapy.

Packer RJ in a review article, "Childhood medulloblastoma: progress and future challenges" (Brain Dev 1999;21:75-81) comments that recent treatment trials have attempted to reduce the amount of craniospinal radiation therapy. In older children, the outcome may be good following reduced-dose CRT with adjuvant chemotherapy. For children with poor risk, intensification of chemotherapy during and after CRT is being explored. Treatment of infants and young children with MED remains a problem.

\section{PRESENTING SYMPTOMS OF NEUROFIBROMATOSIS TYPE 2}

The frequency and mode of presentation of neurofibromatosis type 2 (NF2) was investigated at St Mary's Hospital, the Royal Manchester Children's Hospital, and the Manchester Royal Infirmary, Manchester, UK. A review of records of the Manchester Children's Tumour Registry from 1954 showed 61 (18\%) of 334 patients with NF2 presented in the pediatric age group, at 15 years or less. Of these 61 patients, 26 presented with symptoms of vestibular schwannoma (hearing loss, tinnitus, and facial palsy), while 19 presented with symptoms of a meningioma, 7 with spinal tumor, and 5 with cutaneous tumor. Children of affected parents are at $50 \%$ risk of NF2. Those with no family history probably have a new mutation of the NF2 gene. (Evans DGR, Birch JM, Ramsden RT. Paediatric presentation of type 2 neurofibromatosis. Arch Dis Child Dec 1999;81:496-499). (Respond: Dr DGR Evans, Department of Medical Genetics, St Mary's Hospital, Manchester M13 0JH, UK).

COMMENT. Primary diagnostic criteria for neurofibromatosis type 2 are: Bilateral vestibular schwannomas or family history of NF2 plus 1) Unilateral vestibular schwannoma or 2) Any two of: meningioma, glioma, neurofibroma, 